\title{
Corrigendum: Seed Embryo Development Is Regulated via an AN3-MINI3 Gene Cascade
}

\begin{abstract}
Lai-Sheng Meng ${ }^{1,2 *}$, Yi-Bo Wang ${ }^{3}$, Gary J. Loake ${ }^{2,4 *}$ and Ji-Hong Jiang ${ }^{1,2 *}$
${ }^{1}$ The Key Laboratory of Biotechnology for Medicinal Plant of Jiangsu Province, School of Life Science, Jiangsu Normal University, Xuzhou, China, ${ }^{2}$ Transformational Centre for Biotechnology of Medicinal and Food Plants, Jiangsu Normal University - Edinburgh University, Xuzhou, China, ${ }^{3}$ School of Bioengineering and Biotechnology, Tianshui Normal University, Tianshui, China, ${ }^{4}$ Institute of Molecular Plant Sciences, School of Biological Sciences, Edinburgh University, Edinburgh, United Kingdom
\end{abstract}

Keywords: ANGUSTIFOLIA3 (AN3), MINISEED3 (MINI3), seed mass, embryo cell elongation, embryo cell division, Arabidopsis

\section{A corrigendum on}

Seed Embryo Development Is Regulated via an AN3-MINI3 Gene Cascade by Meng, L.-S., Wang, Y.-B., Loake, G. J., and Jiang, J.-H. (2016). Front. Plant Sci. 7:1645. doi: $10.3389 / f p l s .2016 .01645$

\section{OPEN ACCESS}

Edited and reviewed by: Jin-Gui Chen, Oak Ridge National Laboratory, United States

${ }^{*}$ Correspondence:

Lai-Sheng Meng

meng/sh@jsnu.edu.cn Gary J. Loake

gloake@ed.ac.uk Ji-Hong Jiang

jhjiang@jsnu.edu.cn

Specialty section: This article was submitted to

Plant Cell Biology, a section of the journal Frontiers in Plant Science

Received: 19 May 2017 Accepted: 06 June 2017 Published: 14 June 2017

Citation:

Meng L-S, Wang Y-B, Loake GJ and Jiang J-H (2017) Corrigendum: Seed Embryo Development Is Regulated via an AN3-MINI3 Gene Cascade.

Front. Plant Sci. 8:1073. doi: 10.3389/fp/s.2017.01073
In the original article, there was a mistake in the legend for [Figure 6] as published. [We state the mistake which was made in A, B, H and J. Moreover, we added Bars]. The correct legend appears below. The authors apologize for this error and state that this does not change the scientific conclusions of the article in any way.

In the original article, there was a mistake in [Figure 6] as published. [We state the mistake which was made in G and F]. The corrected [Figure 6] appears below. The authors apologize for this error and state that this does not change the scientific conclusions of the article in any way.

In the original article [Kawade, K., Horiguchi, G., and Tsukaya, H. (2010). Non cellautonomously coordinated organ size regulation in leaf development. Development 137: 42214227] was not cited in the article. The citation has now been inserted in [RESULTS], [an3-4 Plants Have Increased Seed Mass], [Paragraph Number 1] and should read:

an3-4 plants have increased seed mass

While investigating drought tolerance exhibited by an3-4 plants, we observed this line had larger cotyledons than wild-type (Col-0). To confirm whether these large cotyledons were due to a lack of AN3 protein activity, we investigated the phenotype of an3-4 complemented lines (Kawade et al., 2010).

The authors apologize for this error and state that this does not change the scientific conclusions of the article in any way.

In the original article, there was an error. [The mistake that was made in AN3 Expression Pattern in RESULTS].

A correction has been made to [RESULTS], [AN3 Expression Pattern], [Paragraph 8]:

To further explore AN3 function, we examined the expression pattern of the $\beta$-glucuronidase (GUS) reporter gene driven by a native AN3 promoter. Analysis of Arabidopsis transformed with an approximately $2.0-\mathrm{kb}$ AN3 promoter-GUS reporter gene fusion (AN3pro:GUS) showed that AN3pro:GUS was expressed in integument and seed coat (Figures 6A,B), stigmas (Figure 6D), siliques, young leaves and hypocotyls (Figures 6F-H) and embryos (Figure 6J), but not in mature 

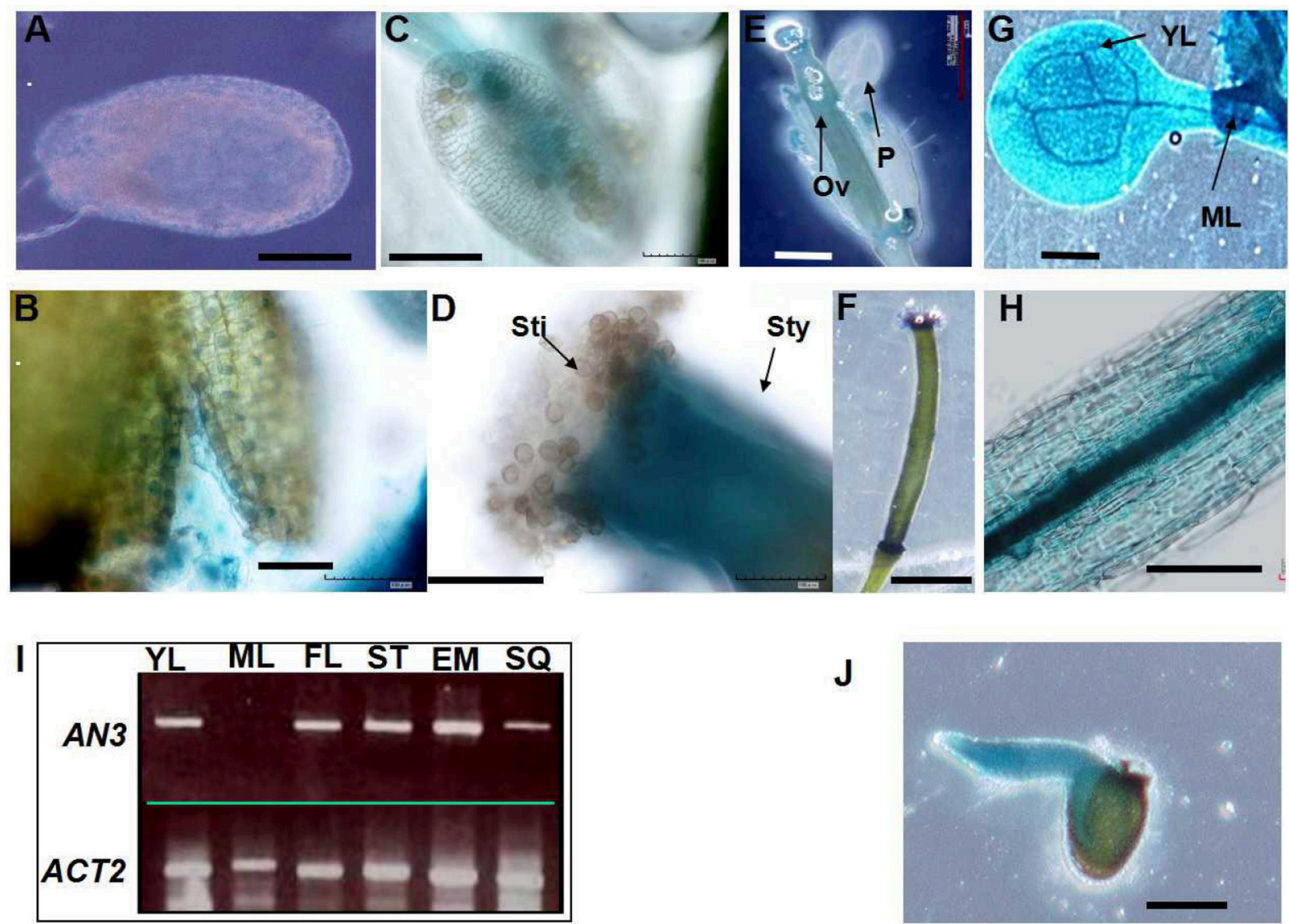

FIGURE 6 | AN3 expression analysis. Expression of AN3pro:GUS in the integument (A), seed coat (B), hypocotyl (H), and embryos (J). Bars = $50 \mu \mathrm{m}$ for $(\mathbf{A}-\mathbf{H})$ and (J).

pollen grains (Figure 6C) petals (Figure 6E), mature leaves (Figure 6G). Consistent with this, RT-PCR results indicated the presence of $A N 3$ transcripts in the corresponding organs (Figure 6I).

In the original article, there was an error. [The mistake that was made in an3-4 Plants Have Increased Seed Mass in RESULTS].

A correction has been made to [RESULTS], [an3-4 Plants Have Increased Seed Mass], [Paragraph 1]:

These lines harboring a 35S:AN3 transgene in an an3-4 genetic background exhibited similar cotyledon size to Col0 (Supplementary Figures S1A,B). A GRF1 loss-of-function mutant showed similar cotyledon size relative to wild-type (Supplementary Figures S1A,B), implying GRF1 may not be involved in the regulation of seed mass. Taken together our data suggests that the an3-4 plants have larger cotyledons relative to wild-type (Col-0).

In the original article, there was an error. [The mistake that was made in The Large Seeds of an3-4 Plants Are Due to Increased Embryo Cell Size in RESULTS].
A correction has been made to [RESULTS], [The Large Seeds of an3-4 Plants Are Due to Increased Embryo Cell Size], [Paragraph 4]:

However, these parameters were similar in grf1 plants relative to wild-type (Figures 3A,C). Cytological observations indicated that the average area of cotyledon embryos from an3-4 plants was about 1.40 times that of wild-type (Figures 3B,D).

The authors apologize for this error and state that this does not change the scientific conclusions of the article in any way.

Conflict of Interest Statement: The authors declare that the research was conducted in the absence of any commercial or financial relationships that could be construed as a potential conflict of interest.

Copyright ( 2017 Meng, Wang, Loake and Jiang. This is an open-access article distributed under the terms of the Creative Commons Attribution License (CC BY). The use, distribution or reproduction in other forums is permitted, provided the original author(s) or licensor are credited and that the original publication in this journal is cited, in accordance with accepted academic practice. No use, distribution or reproduction is permitted which does not comply with these terms. 